EPJ Web of Conferences 16, 01003 (2011)

DOI: $10.1051 /$ epjconf/20111601003

(C) Owned by the authors, published by EDP Sciences, 2011

\title{
Observations of planetary transits at the University Observatory Jena
}

\author{
S. Raetz ${ }^{1, a}$, M. Mugrauer ${ }^{1}$, T.O.B. Schmidt ${ }^{1}$, T. Roell ${ }^{1}$, T. Eisenbeiss ${ }^{1}$, \\ M. Vaňko ${ }^{1}$, A. Koeltzsch ${ }^{1}$, M.M. Hohle ${ }^{1,3}$, Ch. Ginski ${ }^{1}$, C. Marka ${ }^{1}$, \\ M. Moualla ${ }^{1}$, N. Tetzlaff ${ }^{1}$, A. Reithe ${ }^{1}$, W. Rammo ${ }^{1}$, S. Fiedler ${ }^{1}$, \\ J. Koppenhoefer ${ }^{2}$ and R. Neuhäuser ${ }^{1}$
}

${ }^{1}$ Astrophysical Institute und University Observatory Jena, Schillergässchen 2-3, 07745 Jena, Germany

${ }^{2}$ University Observatory Munich, Scheinerstrasse 1, 81679 München, Germany

${ }^{3}$ Max-Planck-Institut of Extraterrestial Physics, Giessenbachstrasse, 85748 Garching,

Germany

\begin{abstract}
We report on observations of transit events of the transiting planets XO-1b, TrES-1 and TrES-2 with a $25 \mathrm{~cm}$ telescope of the University Observatory Jena. With the help of all available transit times from literature including our own photometry our measurements allowed us to refine the estimate of the orbital period of all three transiting planets observed by us.
\end{abstract}

\section{INTRODUCTION}

In this work we use the transit method to observe known transiting planets at the University Observatory Jena. We paid special attention to the accurate determination of transit times in order to identify precise transit timing variations that would be indicative of perturbations from additional bodies ([1]) and to refine the orbital parameters of the systems. First results were presented in [2] and [3].

Here, we present results for XO-1b, TrES-1 and TrES-2 that we obtained from our observations with a $25 \mathrm{~cm}$ telescope at the University Observatory Jena.

\section{OBSERVATIONS}

Most observations were carried out at the University Observatory Jena which is located close to the village Großschwabhausen, $10 \mathrm{~km}$ west of the city of Jena. [4] describes the instrumentation and operation of the system. Our transit observations are carried out with the CTK (Cassegrain Teleskop Kamera), the CCD imager operated at the $25 \mathrm{~cm}$ auxiliary telescope of the University Observatory Jena.

\section{XO-1B}

XO- $1 \mathrm{~b}$ was the first transiting planet observed at the University Observatory Jena. Between March 2007 and May 2009 four transit events were observed. With an exposure time of $60 \mathrm{~s}$ we achieve a mean cadence of the data points of $1.4 \mathrm{~min}$. Our mean photometric precision is $0.007 \mathrm{mag}$. In addition to the

ae-mail: straetz@astro.uni-jena.de

This is an Open Access article distributed under the terms of the Creative Commons Attribution-Noncommercial License 3.0, which permits unrestricted use, distribution, and reproduction in any noncommercial medium, provided the original work is properly cited. 
transits observed at the University Observatory Jena we could find 54 transit times from 2004-2009 in the literature ([5-7], ETD ${ }^{1}$ ). Through timing residuals analysis we find that the orbital period is slightly smaller compared to the previously published period. Thus, our measurement of the transit time lead to a refined estimate of the ephemeris:

$$
T_{\mathrm{c}}(E)=(2453808.91705+E \cdot 3.941501) \mathrm{d} .
$$

\section{TrES-1}

We observed three transits of TrES-1 in front of it's parent star with our $25 \mathrm{~cm}$ Cassegrain telescope. Our mean photometric precision of the $V=11.79$ mag star is 0.009 mag. For TrES- 1 we found 52 midtransit times between 2003 and 2009 in the literature ([8-12], ETD). With all available times of these altogether 55 transits we determined the transit timing residuals for TrES-1. This allowed us to refine the estimate of the ephemeris:

$$
T_{\mathrm{c}}(E)=(2453186.806341+E \cdot 3.0300722) \mathrm{d} .
$$

\section{TrES-2}

Since March 200713 different transits were observed. All TrES-2 observations were taken in I-band with $60 \mathrm{~s}$ exposure time. The mean photometric accuracy of the $\mathrm{V}=11.4$ mag bright TrES-2 host star is $0.007 \mathrm{mag}$. With the transit timings for TrES-2 from 40 events published in literature ([13, 14], ETD) plus our own 13 transits, we found that the orbital period slightly lower $(\sim 0.6 \mathrm{~s})$ compared to the previously published period. The resulting ephemeris which represent our measurements best is:

$$
T_{\mathrm{c}}(E)=(2453957.63492+E \cdot 2.470614) \mathrm{d} \text {. }
$$

\section{SUMMARY AND CONCLUSIONS}

Using our observations at the University Observatory Jena with a $25 \mathrm{~cm}$ Cassegrain telescope equipped with the optical CCD camera CTK we could observe the known planetary transits of XO-1b, TrES-1 and TrES-2. In all three cases our measurement of the transit times lead to a refined estimate of the ephemeris, improved by $0.1 \mathrm{~s}, 0.6 \mathrm{~s}$ and $3 \mathrm{~s}$ for TrES-1, TrES-2 and XO- $1 \mathrm{~b}$, respectively.

The mean photometric precision of the observed stars $(\mathrm{V}=11-12 \mathrm{mag})$ with known transiting planets is $0.008 \mathrm{mag}$ and the precision in the determination of the transit times is $\sim 0.0013 \mathrm{~d}$. This allows us to register transit time variations of around $\sim 2 \mathrm{~min}$.

We will continue observing stars with known transiting planets to search for transit time variations for the next few years to decades, also using a new, better CCD-camera with our $60 \mathrm{~cm}$ Schmidt telescope.

\section{References}

[1] Agol, E., Steffen, J., Sari, R., \& Clarkson, W., MNRAS 359, 567 (2005)

[2] Raetz, S., et al., AN 330, 475 (2009)

[3] Raetz, S., et al., AN 330, 459 (2009)

[4] Mugrauer, M., AN 330, 419 (2009)

[5] McCullough, P. R., et al., ApJ 648, 1228 (2006)

[6] Wilson, D. M., et al. PASP 118, 1245 (2006)

\footnotetext{
${ }^{1}$ Exoplanet Transit Database, http://var.astro.cz/ETD
} 
Research, Science and Technology of Brown Dwarfs and Exoplanets

[7] Holman, M. J., et al. ApJ 652, 1715 (2006)

[8] Alonso, R., et al., ApJ 613, L153 (2004)

[9] Charbonneau, D., et al., ApJ 626, 523 (2005)

[10] Winn, J. N., Holman, M. J., \& Roussanova, A., ApJ 657, 1098 (2007)

[11] Narita, N., et al. PASJ 59, 763 (2007)

[12] Hrudková, M., et al., IAU Symposium 253, 446 (2009)

[13] O’Donovan, F. T., et al., ApJ 651, L61 (2006)

[14] Holman, M. J., et al., ApJ 664, 1185 (2007) 